Cinémas

Revue d'études cinématographiques

Journal of Film Studies

\title{
NINEY, François, L'Épreuve du réel à l'écran. Essai sur le principe de réalité documentaire, Bruxelles, De Boeck Université, 2000, 346 p.
}

\section{Marion Froger}

Volume 12, numéro 3, printemps 2002

Cinélekta 4

URI : https://id.erudit.org/iderudit/000740ar

DOI : https://doi.org/10.7202/000740ar

Aller au sommaire du numéro

Éditeur(s)

Cinémas

ISSN

1181-6945 (imprimé)

1705-6500 (numérique)

Découvrir la revue

Citer ce compte rendu

Froger, M. (2002). Compte rendu de [NINEY, François, L'Épreuve du réel à l'écran. Essai sur le principe de réalité documentaire, Bruxelles, De Boeck Université, 2000, 346 p.] Cinémas, 12(3), 165-173.

https://doi.org/10.7202/000740ar d'utilisation que vous pouvez consulter en ligne. 


\section{N IN EY, François, L'É preuve du réel à l'écran. Essai sur le prin- cipe de réalité documentaire, Bruxelles, De Boeck Université, 2000, $346 \mathrm{p}$.}

\section{Introduction}

Les professeurs et leurs étudiants se réjouiront à juste titre de la parution de L'Épreuve du réel à l'écran. Essai sur le principe de réalité documentaire, ouvrage qui se présente comme un manuel scolaire quelque peu atypique. Les publications des dernières années cherchaient à classifier les documentaires suivant des écoles, des mots d'ordre ou des catégorisations théoriques ${ }^{1}$; à faire l'histoire du documentaire ${ }^{2}$; à théoriser son mode de production de sens; ou encore à offrir une perspective sociologique sur ses modes de réception et de production ${ }^{3}$. Rien de tel chez $\mathrm{N}$ iney, qui déplace la question du documentaire du terrain de la rhétorique du discours à celui de l'esthétique de la réflexion. Pour ce faire, et cela n'est pas le moindre mérite de son propos, N iney nous entraîne dans un vaste tour d'horizon des questions qu'il faut avoir en tête lorsqu'on entreprend l'étude du documentaire, qu'on sinterroge sur l'image documentaire et son rapport à la réalité, sur la dimension artistique de l'analyse du réel ou encore sur le rapport à la vérité impliqué par le mode de réception du film.

L'ouvrage de Niney vaut aussi par la diversité et la richesse de ses références. $O$ n sent qu'il est issu d'une longue fréquentation du documentaire, d'un vivre avec celui-ci, qui lui permet de tisser un vaste réseau de résonances entre des théoriciens, des cinéastes, des écrivains, des philosophes ou des scientifiques (Bazin, Péléchian, Perec, Arendt et $\mathrm{H}$ eisenberg, pour ne citer qu'eux). C'est somme toute le parcours intellectuel d'un homme, de ce qu'il a lu, vu, entendu, enseigné à l'ouvrage mémoriel qui rassemble en une anthologie les trouvailles que lui 
ont fournies moult rencontres, lectures et projets d'écriture en relation avec le documentaire et les documentaristes.

\section{La pédagogie}

On peut sans conteste attribuer une grande valeur pédagogique à l'angle d'attaque proposé par $\mathrm{N}$ iney. L'organisation des chapitres en cinq grandes parties distinctes permet de distinguer: 1) des propositions théoriques sur la «(re)-production du monde ${ }^{4} \gg ; 2$ ) des problématiques traditionnelles d'ordre esthétique (comment l'art peut être un rempart contre la propagande ou la propagation de l'idéologie totalitaire, Leni Riefensthal constituant à cet égard un cas d'école), d'ordre politique (comment la voix off instaure un certain ordre du discours, comme voix de la Raison, de la Nation, du Progrès) et sociohistorique (comment l'image est tributaire d'une Weltanschauung dominante) ; 3) des points stratégiques de la production et de l'exploitation des images (la question du «bon ou mauvais» usage de l'image par les médias de masse) ; 4) des pistes d'analyse des conditions cinématographiques de la mémoire collective et de sa réactivation. Niney use alors d'une opposition fort simple entre «l'actualité» des images journalistiques et les nappes de temps des images documentaires; 5) une réflexion conclusive sur la fausse distinction entre documentaire et fiction qui repose sur une partition trop rigide entre le vrai et le faux, où l'on manque ce qui passe de l'un à l'autre, la fonction de leur interpénétration dans la production de sens.

Au lieu de partir de la fausse évidence d'une réalité prise sur le vif (angle qui amène son lot de questions sur la nature représentationnelle ou testimoniale de l'image), Niney part de la construction du réel par le média cinématographique et télévisuel, que le documentaire comme pratique récupère, conteste, renouvelle, etc. II va ensuite élargir son champ de réflexion en rattachant la question de la vérité des images aux débats contemporains sur le statut de la vérité scientifique. L'intérêt est que le documentaire n'est plus marginalisé comme production discursive de seconde main (relevant de la vulgarisation scientifique ou du média d'opinion dans le meilleur des cas), mais valorisé, comme processus valide de connaissance, à partir de ses proposi- 
tions esthétiques plutôt que de ses qualités discursives. C'est en effet contre une approche «documentarisante» que $N$ iney se dresse, qui place la pertinence du documentaire dans l'effacement de ses traits esthétiques et narratifs. La démarche est dans l'air du temps. Les critères de scientificité des sciences humaines sont aujourd'hui réévalués, non plus en fonction de ceux des sciences dures (il ne sagit plus de se doter des mêmes outils conceptuels, des mêmes protocoles de recherche, des mêmes conditions d'analyse que la physique, la biologie ou les mathématiques), mais à l'aune des puissances de «l'imaginaire»: on insiste davantage sur les possibilités, les formes, les caractéristiques de différents types de construction par la pensée, impliquant des processus de fictionnalisation, de subjectivisation, de visualisation déjà observés dans les pratiques artistiques. $N$ on, les choses ne sont pas ce qu'elles sont (N iney évoquera alors la «vérité tensionnelle» que Paul Ricœur expose dans La M étaphore vive). Par l'indétermination du langage, $N$ iney nous emmène tranquille ment jusqu'aux pentes de l'art, pour prouver, avec l'argument du documentaire, combien l'art est fondé dans sa prétention de connaissance.

C'est pourquoi il convient de prendre très au sérieux les versions et visions alternatives, les «manières de faire des mondes» (N elson Goodman) que peuvent offrir les documentaires dits de création. Attendu que création ne s'oppose pas à connaissance, pas plus qu'invention à réalité (p. 246).

\section{«Essai sur le principe de réalité documentaire 》}

Un tel sous-titre nous en dit long sur l'ambition philosophique de Niney. II sagit en fait de prendre prétexte d'une étude sur le documentaire pour relancer le débat sur le concept de réalité. L'angle d'approche est familier aux Cultural Studies, puisque le concept de réalité est analysé à travers l'histoire du partage entre l'objectif et le subjectif, qui se lit directement dans la production documentaire.

Le rapport film/filmeur/spectateur devient ainsi un analyseur non seulement esthétique mais politique, de ce que l'on considère, accepte ou conteste comme la réalité à telle période, c'està-dire du partage entre subjectif et objectif (partage au double 
sens de diviser et de mettre en commun). Si la réalité nous pré existe, nous détermine et nous résiste, notre présence et nos liens la transfigurent, la restituent transformée à nos contemporains et héritiers ( $p .9)$.

C'est là une des pistes de réflexion les plus stimulantes qu'ouvre le livre, car elle fait de l'étude du documentaire un moyen d'aborder des questions proprement épistémologiques. II n'y a pas de contenu objectif attribuable au concept de réalité, mais une épaisseur de sens correspondant à ses différents moments historiques. L'idée n'est pas nouvelle, mais la preuve, développée par N iney à partir du documentaire, est originale et convaincante. Cependant, $\mathrm{N}$ iney suit une pente quelque peu hasardeuse quand il tente d'imposer une conception du réel en croisant les réflexions similaires de cinéastes, de philosophes et de scientifiques. L'impression fâcheuse qui en ressort est qu'il plaque sur le fait documentaire de nouveaux dogmes philosophiques et scientifiques, faisant du premier l'illustration des derniers. $N$ iney fait maladroitement de la mise en cause de la notion d'objectivité son point de départ et son point d'arrivée. Le problème est tout d'abord dans cette boucle: parti de la remise en cause de l'objectivité (le monde comme représentation), il conclut sur la remise en cause de l'objectivité, comme si aucun chemin n'avait été finalement parcouru, comme si on ne faisait que déboucher finalement sur ce savoir supposé acquis au début... Le problème n'est pas simplement méthodologique: le documentaire n'apparaît plus que comme un instrument au service d'un rabâchage critique stérile.

Reste au lecteur une synthèse fort bien menée sur la question du statut du réel. II jugera alors que le documentaire est fort bien outillé pour assumer la démarche critique que Cassirer définit comme suit (p. 10): le «glissement dans le subjectif » de ce qui avait la stabilité de l'objectif (et que l'on tenait donc pour connaissance acquise) n'affecte pas les choses elles-mêmes, mais concerne plutôt «l'évaluation critique des connaissances» (I'objectivité qui se défait est le résultat de cette évaluation critique). Le lecteur éclairera sa compréhension du documentaire à la lumière de ce que $\mathrm{H}$ annah Arendt affirme du rapport entre propagande et vision de la réalité: il y aurait une vision de la réalité propre 
aux masses, qui refuse son caractère «fortuit», préfère la cohé rence du réel à la simple coïncidence des faits. $\mathrm{H}$ annah Arendt dénonce une fictionalisation du réel dans la propagande qui correspond à un besoin des masses à un moment donné: besoin de condamner le monde [réel] «dans lequel elles sont contraintes de vivre et ne peuvent subsister »(p. 70). Enfin, le lecteur appréciera sans doute le rappel des thèses d'H eisenberg, qui permet à $\mathrm{N}$ iney de dénoncer le dogme de l'objectivité. En premier lieu, au chapitre XII, il s'attaque au réductionnisme logique et tente de faire ressortir la part irrationnelle du réel. II dénonce alors la réduction du langage à de l'information, qui nécessite un ensemble de définitions fixes correspondant à des objets donnés, et la réduction du fonctionnement du cerveau au fonctionnement d'un ordinateur $^{5}$. Puis, dans le chapitre XIV, Niney s'en prend directement au dogme de l'objectivisme. II commence par un plaidoyer efficace contre les pratiques télévisuelles de l'information puis sattaque aux notions de vrai et de faux avec le soutien de H eisenberg $^{6}$ : ce n'est jamais la relation au réel qui ultimement décide de ce qui est exact ou faux, cela se décide au mieux à l'intérieur de l'idéalisation qui a médiatisé notre rapport au réel : "on doit donc s'accommoder du fait que ce n'est qu'à travers le processus de connaissance lui-même que se décide ce qu'on doit entendre par "connaissance" » (p. 238). N iney part donc de la religion de l'image objective - qui neutralise le processus de production de cette objectivité - pour aller vers la nécessité du montage comme processus de connaissance du réel (condition d'une prise sur lui). Q uelle connaissance la télévision produit-elle? Tout au plus un sociologisme de bas étage insiste $\mathrm{N}$ iney:

$D$ ans les bonnes expériences scientifiques, de la mise en évidence du comment cela se passe, jaillit le pourquoi ça se passe comme ça. [... ] II ne s'agit pas de décrire des phénomènes, mais de leur faire rendre raison. [... ] $D$ ans sa reproduction classifiée de l'actuel, la télévision relève du sociologisme de bas étage, comptabilisant les faits, catégorisant les opinions. Les techniques du direct sont recrutées ici pour attester la présence de la caméra sur les lieux, l'évidence de l'événement, sa vérité instantanée; là pour échantillonner à coup de microstrottoirs et de micros-cravates l'opinion publique et 
celle de ses représentants. O $\mathrm{r}$ l'enjeu du documentaire, c'est au contraire d'arriver à voir autre chose que ce qui est déjà vu (p. 243).

Le gain d'une telle approche est loin d'être négligeable: elle permet de dissiper l'illusion induite par notre rapport «médiatique» à l'image en dénonçant les nouveaux sophistes du visuel, à savoir ceux qui nous font croire à l'objectivité de la réalité pour nous imposer comme unique la vérité de leur image. La charge est convaincante même si elle est un peu laborieuse (l'ouvrage compte 346 pages). N iney nous démontre ainsi les conséquences désastreuses pour la pensée d'une croyance aveugle en la vérité des images. A près avoir déraciné l'arbre télévisuel qui nous cachait la forêt des images documentaires, les potentialités de celles-ci peuvent être redécouvertes. Ces potentialités, N iney nous les présente à travers une nouvelle définition de notre rapport au monde, inspirée d'H eisenberg:

Car, n'en déplaise à l'indifférente objectivité médiatique, nous ne sommes plus les spectateurs d'un monde à déchiffrer mais acteurs du théâtre de la vie. Pour le dire encore avec $\mathrm{H}$ eisenberg, partout, dans la nature qu'il s'est réappropriée par la technique, «[I]'homme ne rencontre plus que lui-même» et paradoxalement se retrouve perdu, car il touche aux limites du progrès technique, non pas quantitativement, mais quant au dévoilement du sens. Moment de retournement où I'homme, qui croyait trouver le sens des choses dans les choses, se découvre indissolublement producteur du sens des choses dans le cadre de systèmes de pensée limités et de cohabitation relatifs.

D ans cette situation nouvelle, "sans partenaire ni adversaire», I'homme se trouve en demeure de renoncer à l'idéal de maitrise et de vérité absolue, d'inventer de nouvelles valeurs «dans un espace à un plus grand nombre de dimensions» et de forger un nouveau concept de réalité (qui ne saurait plus figurer une totalité promise et soumise, mais une connexion de champs d'interactions et d'interrogations partiels) (p. 317). 


\section{Conclusion}

$\mathrm{N}$ iney assoit finalement ses propositions philosophiques sur le socle du documentaire. La vérité, peut-il alors affirmer, est «justesse esthétique»:

On vous montre le monde tel qu'il est et il est comme on vous le dit. Pas d'erratum. C'est contre ce sens unique, contre l'image adéquate et suffisante que s'inscrivent en faux les grands documentaristes quej'ai convoqués. Chacun à sa manière a inventé de nouvelles relations du et au réel, déplacé la frontière entre objectif et subjectif, ouvert dans le réel des brèches de doute et de possible par les jeux décalés du dire et du voir (p. 10-11).

Cette position implique de croire à la puissance individuelle de l'imagination, de dénoncer les modes cartésiens de reconnaissance de la vérité (ce qui se manifeste avec évidence, ce qui n'est pas contradictoire, ce qui ne souffre aucun doute) et de les remplacer par de nouvelles val eurs déstabilisantes comme la mise en doute et la pratique de l'écart, autant de valeurs dites «esthétiques» parce que plus ou moins héritées du romantisme. Cependant, la question du rapport entre l'image et la pensée reste en quelque sorte biaisée par le parti pris de départ, à savoir de se prévaloir de la seule analyse esthétique comme angle d'approche. Le lecteur de N iney reste sur sa faim. L'«essai » annoncé ne va pas au bout de son contrat, sil en avait un, de recherche. $\mathrm{Car}$ contrairement à ce qu'a fait $D$ eleuze dans L'I mage mouvement et L'I mage temps, N iney ne réinterroge pas la pensée à l'aune de la pratique documentaire; il se contente de nous expliquer comment il convient de le voir et de l'écouter pour en apprécier la pertinence. Au terme de ce livre, point de découvertes majeures donc, sur la pensée elle-même (ni sur le principe de réalité), mais un palmarès des réussites et des échecs qui émerge de la démarche toute pédagogique de $\mathrm{N}$ iney, consistant, pour mieux dégager son point de vue, en des comparaisons de choix esthétiques et de manières de faire.

Niney procède en avocat de la cause documentaire, comme en atteste son vocabulaire et ses méthodes. II reprend $\mathrm{H}$ eisenberg pour montrer comment le documentaire correspond 
parfaitement à sa conception de la production de savoirs: «il y a des agencements idéalisés de régions de réalité. »C ertes, d'un point de vue pédagogique, il peut être intéressant de détruire de fausses conceptions sur la vérité et la réalité, produites par une méconnaissance des méthodologies et des conditions de validité de la pensée scientifique (la première, somme toute, à qui on demande de rendre des comptes sur le réel). O n demeure cependant sceptique: prouver le bien-fondé d'une démarche artistique par la caution des interrogations scientifiques a déjà laissé de tristes souvenirs de récupération métaphysique.

Ainsi, il est clair que, si N iney ne part pas de l'opposition documentaire/fiction, sa pensée n'en est pas moins dichotomique puisqu'il oppose, à chaque chapitre de son ouvrage pour ainsi dire, la production des médias de masse audiovisuels au véritable cinéma documentaire (qu'il défend dans son ensemble, au-delà des distinctions d'écoles) qui pratique «des découpages/montages de la réalité assumant une dynamique de la contradiction, une profondeur du temps, des usages de la métaphore, des dispositifs d'interlocution ou d'anthropologie partagée (Rouch, M arker, W iseman, Van der Keuken, Robert K ramer) ». Là encore, on peut s'interroger sur les véritables moyens dont dispose le documentaire pour émanciper ses images du joug du flux télévisuel. D ans quelle mesure des interventions «créatrices» jouent-elles vraiment quand le sens des images dépend au moins tout autant du contexte de leur réception? Cette question reste en suspens, et nécessiterait sans doute un tout autre ouvrage...

M arion Froger

Université de M ontréal

\section{NOTES}

1. À partir d'une détermination toute négative (le fameux non-fiction film des Anglo-Saxons), on a distingué des catégories (actualités, films pédagogiques, films ethnographiques, etc.) ou des écoles (école russe du ciné-œil, école canadienne du Candid Eye, école québécoise du cinéma direct, etc.), des fonctions politiques ou scientifiques (conscientisation, éducation populaire, propagande, archivage de données, etc.).

2. Le parcours historique est quand même très présent, notamment dans la deuxième partie intitulée «Du parlé au parlant»: N iney y fait l'histoire de l'institu- 
tionnalisation du documentaire à travers son instrumentalisation sociopolitique comme média de masse, ce qu'il appelle sa fonction de «prévision du monde».

3. Le documentaire, dans les études cinématographiques, fait figure de parent pauvre: peu d'ouvrages lui sont consacrés; historiens et théoriciens ont toujours mis davantage l'accent sur des questions qui intéressent avant tout la fiction cinématographique: des questions de narratologie (propriétés et fonctions du récit cinématographique), de sémiologie (la production de sens comme fait langagier, à partir de la fonction narrative) ou d'esthétique (pouvoir de l'image comme création) ont surtout été traitées; des méthodes d'investigation historique ont poussé les historiens à considérer le film comme un phénomène social complexe en tant que spectacle, marchandise, industrie, média, etc., mais la tendance semble sinverser de nos jours, sous la pression du monde virtuel. O n n'affronte plus le réel à travers la question de ses représentations (images photographiques, cinématographiques, numériques, etc.) mais, en mettant le réel en perspective comme construction, on tente d'identifier les principaux agents de son dévoilement, dans les médias modernes qui articulent l'expérience et les souvenirs. Bref, la question du documentaire est considérablement renouvelée par ce type d'approche.

4. N iney explique en tête de cette partie: «L'apparition du cinéma jette le trouble dans l'ordre des représentations, en confondant l'enregistrement objectif et le regard subjectif, la science et l'art, le réel et la fiction. En transposant le monde sans avoir l'air d'y toucher et en diffusant partout des images vivantes, le cinéma est l'avantcoureur d'un nouveau signe du temps: I'actualité» (p. 20). O n notera que la collusion de ces deux assertions produit en quelque sorte le malentendu documentaire, à savoir qu'il existe une «présentation » possible du présent, qui fasse l'économie de la confusion de l'objectif et du subjectif propreà toute représentation.

5. N iney cite Edelman : «La pensée ne se limite pas à la manipulation de symboles abstraits dont la signification est justifiée par référence univoque à des choses réelles» (p. 194).

6. D ont il rappelle pertinemment l'idée maîtresse qu'il y a plusieurs régions de réalité suivant les «agencements» que nous opérons. On comprend l'intérêt cinématographique de cette pensée en relevant les termes d'agencement et d'état de choses qui lui servent à décrire le réel connaissable (que l'on retrouvera chez D eleuze aussi, mais dans un tout autre sens) : trois états de choses, trois niveaux de réalité, produits indépendamment de nos modes de connaissance, produits en connexion avec nos processus de connaissance ou encore créés par nos modes de connaissance. 